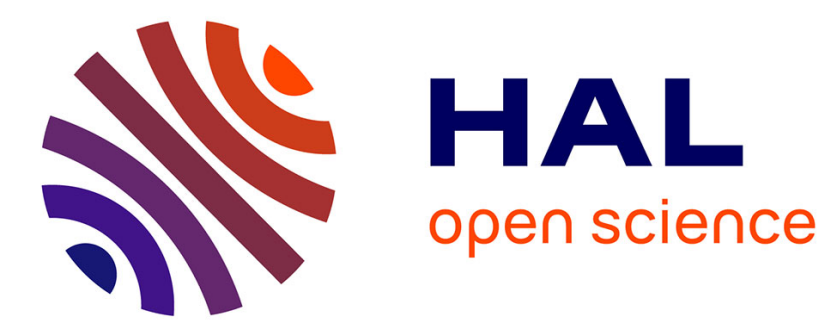

\title{
A damage model based on Kelvin eigentensors and Curie principle
}

\author{
Marc L.M. François
}

\section{To cite this version:}

Marc L.M. François. A damage model based on Kelvin eigentensors and Curie principle. Mechanics of Materials, 2012, 44, pp.23-24. 10.1016/j.mechmat.2011.07.017 . hal-01004697

\section{HAL Id: hal-01004697 https://hal.science/hal-01004697}

Submitted on 29 Jan 2019

HAL is a multi-disciplinary open access archive for the deposit and dissemination of scientific research documents, whether they are published or not. The documents may come from teaching and research institutions in France or abroad, or from public or private research centers.
L'archive ouverte pluridisciplinaire HAL, est destinée au dépôt et à la diffusion de documents scientifiques de niveau recherche, publiés ou non, émanant des établissements d'enseignement et de recherche français ou étrangers, des laboratoires publics ou privés. 


\title{
A damage model based on Kelvin eigentensors and Curie principle
}

\author{
Marc L.M. François \\ Univ Paris-Sud, UPMC Univ Paris 06, CNRS, Lab FAST, Bat 502, Campus Univ, Orsay F-91405, France
}

\begin{abstract}
A general anisotropic damage model is developed that accounts for the thermodynamics of irreversible processes in the framework of generalized standard materials and Kelvin tensor decomposition. Damage is described by fourth-rank tensors, one per eigenspace of the initial stiffness tensor. Their number thus ranges from two for an initially isotropic material to six for an initially triclinic material. The yield criteria are expressed in terms of a limiting energy for each eigenspace. The second-rank eigentensors (at most six) of the fourth-rank damage tensors define the direction of influence of the damage, while the associated eigenvalues characterize its intensity. These eigentensors evolve during loading, inducing an evolution of the symmetry group of the elastic tensor subject to the constraints of the Curie principle.
\end{abstract}

\section{Introduction}

\subsection{Damage mechanics}

The damage concept was introduced by Kachanov (1958) as a decay of the material elasticity under plastic work. Lemaitre (1996) gave a major contribution to the formulation of damage under the framework of the generalized standard materials which was introduced by Halphen and Nguyen (1975) to describe the non linear evolution of solids within the Thermodynamics of Irreversible Processes. Damage processes concern various classes of materials. In particular, the quasi-brittle materials (stone, concrete, ceramic etc). Bazant and Planas (1998) are considered to present only this kind of evolution at early stages of degradation.

At the micro level, damage is associated with the creation of voids or micro-cracks. Micro-mechanical models are based on a homogenization of the behavior of a microscopic cell which considers mechanisms of defects creation and propagation. From pioneer work of Bazant (1984) and Andrieux et al. (1986), this approach led to various models

E-mail address: marc.francois@u-psud.fr
(Chaboche, 1988; Raous et al., 1998; Pensée et al., 2002; Carpinteri et al., 2003; Mattei et al., 2007). However, the complexity of the mechanisms at the micro scale such as grain de-bonding, microcrack sliding and induced opening (Royer-Carfagni and Salvatore, 2000) requires a priori simplifications which induce specific directions to damage, and influences the symmetry group of the elastic tensor of the damaged material.

Another class of models circumvents these difficulties by the use of mathematical analysis of the fourth-rank elasticity tensor which is generally anisotropic (initially and/or due to damage evolution). The decomposition of Boehler et al. (1994) is successfully used in damage mechanics for example by Onat (1984), and Halm and Dragon (1998). The other available decomposition of the elasticity tensor is the Kelvin decomposition which is used in this article.

According to Zheng and Boehler (1994), the symmetry group of the material (its microstructure) is included in the symmetry group of the physical properties (elasticity in our case). For example, according to the (Hermann, 1934) theorem, a composite with pentagonal cells (symmetry group $D_{5}$ ) presents a transverse isotropic linear elasticity (He and Zheng, 1996; Auffray et al., 2009). This is 
consistent with the Curie principle: the symmetry group of the consequences (the elasticity tensor) includes the symmetry group of the causes (the microstructure). Although informations on microstructure can be described by the use of structure tensors (Xiao et al., 2006), we suppose in this introductory article that the symmetry group of the material is also the symmetry group of the elasticity tensor.

\subsection{The Kelvin decomposition}

In its original form it stated the existence of six stresses for which the corresponding strains are proportional, for any linear elastic material (Thomson (Lord Kelvin), 1856). This outstanding result, obtained before tensor analysis, has only been fully understood and rewritten in a modern form (the diagonalization of the elasticity tensor) one century later (Ostrosablin, 1984; Annin and Ostrosablin, 2008; Rychlewski, 1984; Mehrabadi and Cowin, 1990; Helbig, 1994). This decomposition, which expresses the elastic tensor as a collection of weighted (by the Kelvin elastic moduli) fourth-rank projectors, is briefly recalled in Section 2. The proportional projections of the stresses and strains are referred to as eigenstresses and eigenstrains. The Kelvin decomposition allows formulations which are equivalent in stress, strain and energy, suppressing the $a$ priori choice between them (or equivalently between stiffness and compliance) which is generally required. For example, the widely used von Mises criterion belongs to this class as it can be equivalently regarded as the norm of the deviatoric part of the stress or the strain which is an eigentensor of the isotropic elasticity tensor; the Drucker-Prager criterion used in soil mechanics corresponds also to a Kelvin decomposition of the isotropic elasticity. More recently, anisotropic yield surfaces have been developed in the Kelvin framework (Schreyer and Zuo, 1995; François, 1995; Arramon et al., 2000; Desmorat, 2009).

The "basic idea" for the use of the Kelvin decomposition in damage mechanics models consists in the decay of the Kelvin moduli. However, such approach keeps the projectors (thus the symmetry group of the elastic tensor) unchanged. On the contrary, the decay of stiffness can affect tensorial directions which are dependent also upon the applied stress or strain. For example, a damage induced by a tension may leave the response of the material under some hydrostatic pressure or shearing unchanged. The Kelvin decomposition induces naturally the eigenstrain or eigenstress as tensorial direction of damage in case of proportional loading. In case of non proportional loading this direction evolves and the few attempts in the field (Schreyer, 1995; François, 1995) were not fully defined in the general case.

\subsection{The Curie principle}

The Curie principle (Curie, 1894) (for an English translation see Rosen, 1982) postulates that "Lorsque certaines causes produisent certains effets, les éléments de symétrie des causes doivent se retrouver dans les effets produits." which can be translated in "When some causes produce certain effects, the symmetry elements of the causes must be retreived in the effects". Abundant literature can be found on the topic: see for example Chalmers (1970), Sivardière (1995), and Rosen (1995) who stated the symmetry principle as "The symmetry group of the cause is a subgroup of the symmetry group of the effect".

This principle a priori fails in many cases of non linear phenomena. In the classical example of a cylindrical bar buckling under compression the symmetry group of the effects (a reflection with respect to the median plane and a rotation of $\pi$ ) does not includes the one of the causes (the transverse isotropy around the bar axis). Renaud (1935) (see Rosen, 1982 for the English translation) generalizes the Curie principle as follows: "Si un ensemble de causes est invariant par rapport á une transformation quelconque, l'ensemble de leurs effets est invariant par rapport á la même transformation" which corresponds to "If a set of causes is invariant with respect to some transformation, the set of effects is invariant with respect to the same transformation". The concept of set is a key point: in the example of buckling, the bar has as many chances to curve towards one side as towards the others thus the symmetry group of the consequences (envisaged as the set of solutions) includes (in this case, is equal to) the symmetry group of the causes. Similar consideration can be found in the book of Rosen (1995) (see for example p. 85, about the Rutherford experiment).

In the present case of damage mechanics, we consider a large number of microcracks (Lemaitre, 1996). In the simple case of compression, the crack normal forms an angle with the compression axis which can range from $\pi / 4$ according to the Tresca criterion to $\pi / 2$ according to the maximal principal strain criterion (Mazars and PijaudierCabot, 1996) or in between according to other localization criterions (François, 2007). As for the buckling example, this constitutes locally a symmetry breaking. However, the cracks statistically occur at any angle around the axis thus the set of normals form a cone whose axis is the compression axis and whose symmetry group is transverse isotropic as is the compression. In this article we assume the statistical argument is valid thus the Curie's Principle applies.

In damage mechanics the consequence is, at the macro scale of interest, the loss of stiffness of the material. The causes are the actual elasticity, stress and strain tensors. These element have, in general, different symmetry groups. However, the Kelvin decomposition allows us to consider the manifold constituted by the eigentensors which depends upon both the material stiffness and the loading and does not require a choice between stress or strain formulation.

\subsection{This article}

The Kelvin decomposition is recalled in Section 2. The damage model is presented in Section 3. The damage is described by fourth-rank tensors $\mathbb{D}^{i}$ (one by eigenspace $\mathcal{T}^{i}$ ), which are associated with thermodynamic forces $\mathbb{Y}^{i}$ through the Gibbs free energy. According to the generalized standard material framework, the evolution is described by both the normality rule and the consistency equation. Positiveness of the intrinsic dissipation is 
verified. The Kelvin decomposition of the tensors $\mathbb{D}^{i}$ is studied in Section 4. It is shown that the second-rank eigentensors of $\mathbb{D}^{i}$ indicate the damage tensorial direction of influence and that the corresponding eigenvalues define the damage intensity, in the manner of the classical damage theory. Section 5 is dedicated to the verification of the Curie principle. Examples consider initially isotropic material (for sake of simplicity). The first loading path consists in two successive proportional loadings (oedometric tension and shear). It allows a simple comprehension of the model. The two last ones are circular non proportional paths in the same bi-dimensional strain space. They illustrate the path dependance of the model.

\section{The Kelvin decomposition}

This section recalls the Kelvin decomposition of the elasticity tensor and details the notation used in the article. Details can be found in the article of Mehrabadi and Cowin (1990).

The Cauchy stress $\boldsymbol{\sigma}$ and the infinitesimal strain $\boldsymbol{\varepsilon}$ own the index symmetry $\sigma_{\mathrm{ij}}=\sigma_{\mathrm{ji}}$ and $\varepsilon_{\mathrm{ij}}=\varepsilon_{\mathrm{ji}}$ in the canonical basis $\mathbf{e}^{i} \otimes \mathbf{e}^{j}$. A basis $\mathbf{B}^{i}$ of the group $\mathcal{T}$ of the second-rank symmetric tensors can be defined from the orthonormal vector basis $\left(\mathbf{e}^{1}, \mathbf{e}^{2}, \mathbf{e}^{3}\right)$ as:

$$
\begin{aligned}
& \mathbf{B}^{1}=\mathbf{e}^{1} \otimes \mathbf{e}^{1}, \\
& \mathbf{B}^{2}=\mathbf{e}^{2} \otimes \mathbf{e}^{2}, \\
& \mathbf{B}^{3}=\mathbf{e}^{3} \otimes \mathbf{e}^{3}, \\
& \mathbf{B}^{4}=\left(\mathbf{e}^{2} \otimes \mathbf{e}^{3}+\mathbf{e}^{3} \otimes \mathbf{e}^{2}\right) / \sqrt{2}, \\
& \mathbf{B}^{5}=\left(\mathbf{e}^{3} \otimes \mathbf{e}^{1}+\mathbf{e}^{1} \otimes \mathbf{e}^{3}\right) / \sqrt{2}, \\
& \mathbf{B}^{6}=\left(\mathbf{e}^{1} \otimes \mathbf{e}^{2}+\mathbf{e}^{2} \otimes \mathbf{e}^{1}\right) / \sqrt{2} .
\end{aligned}
$$

In this basis the stress $\boldsymbol{\sigma}$ has six components obtained by the double index contraction $\hat{\sigma}^{i}=\boldsymbol{\sigma}: \mathbf{B}^{i}=\sigma_{\mathrm{pq}} B_{\mathrm{pq}}^{i}$. Please note that, in this article, the Einstein's summation convention only applies for lower (straight) indexes, related to the canonical basis. The Euclidean norm is (simply) $\|\boldsymbol{\sigma}\|=\left(\sum_{i=1}^{6}\left(\hat{\sigma}^{i}\right)^{2}\right)^{1 / 2}=\sqrt{\sigma_{\mathrm{pq}} \sigma_{\mathrm{pq}}}$. Similar results are obtained for the strain. This basis is orthonormal i.e. $\mathbf{B}^{i}: \mathbf{B}^{j}=\delta_{i j}$ where $\delta$ is the Kronecker delta.

The fourth-rank elasticity (or Hooke) tensor $\mathbb{C}$ defines the stress to strain relationship in linear elasticity $\boldsymbol{\sigma}=\mathbb{C}: \boldsymbol{\varepsilon}$ equivalent to $\sigma_{\mathrm{ij}}=C_{\mathrm{ijk} \mathrm{l}} \varepsilon_{\mathrm{kl}}$. The index symmetries $\sigma_{\mathrm{ij}}=\sigma_{\mathrm{ji}}$ and $\varepsilon_{\mathrm{kl}}=\varepsilon_{\mathrm{lk}}$ lead to the relations $C_{\mathrm{ijkl}}=C_{\mathrm{jikl}}=C_{\mathrm{ijlk}}$ which allow one to use the basis formed by the 36 terms $\mathbf{B}^{i} \otimes \mathbf{B}^{j}$. The components of $\mathbb{C}$ in this basis are denoted as $\widehat{C}_{i j}=\mathbf{B}^{i}: \mathbb{C}: \mathbf{B}^{j}$. Due to the major index symmetry $C_{\mathrm{ijkl}}=C_{\mathrm{klij}}$ (which is due to thermodynamics considerations), the matrix of components $\widehat{C}_{i j}$ is a $6 \times 6$ symmetric matrix with only 21 independent terms. If the material is stable, $\widehat{C}_{i j}$ is positive definite, invertible, and the compliance tensor $\S$ has the components $\widehat{S}_{i j}=\left(\widehat{C}_{i j}\right)^{-1}$ in the same basis. The diagonalization of this matrix gives the $I \leqslant 6$ eigenvalues $\lambda^{i}$ referred to as the Kelvin moduli and the six eigenvectors $\mathbf{E}^{\text {in }}$ referred to as eigentensors. The Hooke tensor can be expressed as:
$\mathbb{C}=\sum_{i=1}^{I} \lambda^{i} \sum_{n=1}^{N^{i}} \mathbf{E}^{i n} \otimes \mathbf{E}^{i n}$,

where $N^{i}$ is the multiplicity of the corresponding $\lambda^{i}$ and $\sum_{i=1}^{I} N^{i}=6$. This relationship exhibits the fourth-rank projectors $\mathbb{P}^{i}$ :

$\mathbb{P}^{i}=\sum_{n=1}^{N^{i}} \mathbf{E}^{i n} \otimes \mathbf{E}^{i n}$,

which constitute an orthogonal decomposition of the fourth-rank identity tensor:

$\llbracket=\sum_{i=1}^{I} \mathbb{P}^{i}$,

whose components are $\widehat{I}_{i j}=\delta_{i j}$ in the basis $\mathbf{B}^{i} \otimes \mathbf{B}^{\mathrm{j}}$ and $I_{\mathrm{ijkl}}=$ $\left(\delta_{\mathrm{ik}} \delta_{\mathrm{jl}}+\delta_{\mathrm{il}} \delta_{\mathrm{jk}}\right) / 2$ in the canonical basis. As a consequence, the Hooke's law is written in a very synthetic form:

$\mathbb{C}=\sum_{i=1}^{I} \lambda^{i} \mathbb{P}^{i}$

The projectors $\mathbb{P}^{i}$ define the subspace $\mathcal{T}^{i} \in \mathcal{T}$ (of basis $\left\{\mathbf{E}^{i 1} \ldots \mathbf{E}^{i N^{i}}\right\}$ ) and, from the orthogonal decomposition (Eq. 4), we have $\mathcal{T}=\bigoplus_{i=1}^{I} \mathcal{T}^{i}$. The Hooke law is rewritten simply as a set of proportionality relations between the projections of the stress and the strain:

$\boldsymbol{\sigma}^{i}=\lambda^{i} \boldsymbol{\varepsilon}^{i}$

$\boldsymbol{\sigma}^{i}=\mathbb{P}^{i}: \boldsymbol{\sigma}, \quad \boldsymbol{\varepsilon}^{i}=\mathbb{P}^{i}: \boldsymbol{\varepsilon}$,

$\boldsymbol{\sigma}=\sum_{i=1}^{I} \boldsymbol{\sigma}^{i}, \quad \boldsymbol{\varepsilon}=\sum_{i=1}^{I} \boldsymbol{\varepsilon}^{i}$

Due to the orthogonality of these projections, the Helmholtz free energy (from which the stress expression derivates) splits in subparts associated with the free energy of each modes:

$2 \rho \Psi(\boldsymbol{\varepsilon})=\sum_{i=1}^{I} \lambda^{i}\left(\boldsymbol{\varepsilon}^{i}\right)^{2}=\sum_{i=1}^{I} \frac{1}{\lambda^{i}}\left(\boldsymbol{\sigma}^{i}\right)^{2}$.

The positiveness of the free energy for any strain yields $\lambda^{i}>0$. Rychlewski (1984) showed that the symmetry group of the stiffness tensor is the intersection of the symmetry group of its projectors:

$\mathcal{G}(\mathbb{C})=\cap_{i=1}^{I} \mathcal{G}\left(\mathbb{P}^{i}\right)$.

For an isotropic material the Kelvin decomposition gives two isotropic subspaces named respectively $\mathcal{T}^{\mathrm{H}}$ for the uni-dimensional hydrostatic one and $\mathcal{T}^{\mathrm{D}}$ for the 5-dimension deviatoric one:

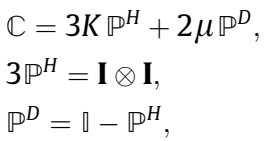

where $K$ is the bulk modulus and $\mu$ the shear modulus. In the basis $\mathbf{B}^{i} \otimes \mathbf{B}^{j}$ these isotropic projectors write as: 
$\begin{aligned} 3 \mathbb{P}^{H} & =\left[\begin{array}{llllll}1 & 1 & 1 & 0 & 0 & 0 \\ 1 & 1 & 1 & 0 & 0 & 0 \\ 1 & 1 & 1 & 0 & 0 & 0 \\ 0 & 0 & 0 & 0 & 0 & 0 \\ 0 & 0 & 0 & 0 & 0 & 0 \\ 0 & 0 & 0 & 0 & 0 & 0\end{array}\right], \\ 3 \mathbb{P}^{D} & =\left[\begin{array}{cccccc}2 & -1 & -1 & 0 & 0 & 0 \\ -1 & 2 & -1 & 0 & 0 & 0 \\ -1 & -1 & 2 & 0 & 0 & 0 \\ 0 & 0 & 0 & 3 & 0 & 0 \\ 0 & 0 & 0 & 0 & 3 & 0 \\ 0 & 0 & 0 & 0 & 0 & 3\end{array}\right] .\end{aligned}$

\section{The proposed anisotropic damage model}

Damage consists, at the macro-scale, in the reduction of the elastic moduli. The simplest way to combine it with the Kelvin decomposition is the constitutive law $\boldsymbol{\sigma}^{i}=$ $\lambda^{i}\left(1-d^{i}\right) \varepsilon^{i}$ in which a scalar damage term $d^{i}$ affects the Kelvin elastic modulus $\lambda^{i}$ related to the elastic projector $\mathbb{P}^{i}$ (Ladevèze, 1993; François, 1995). However such model considers the symmetry group of the material as fixed, independent with respect to the stress or strain symmetry. The presented model is less constraint: the damage effect is described by fourth-rank damage tensors $\mathbb{D}^{i}$ whose evolution is given by the thermodynamics of irreversible processes. The remaining constraint is that $\mathbb{D}^{i}$ act onto the subspace $\mathcal{T}^{i}$ associated with the projector $\mathbb{P}^{i}$.

\subsection{State}

The $I$ fourth-rank damage tensors $\mathbb{D}^{i}$ define, together with the strain $\varepsilon$, the state variables. Each one is associated with an elastic projector $\mathbb{P}^{i}$ (related to the sound material by Eq. 5). The proposed Gibbs free energy is:

$2 \rho \Psi\left(\boldsymbol{\varepsilon}, \mathbb{D}^{i}\right)=\boldsymbol{\varepsilon}: \sum_{i=1}^{I} \lambda^{i}\left(\mathbb{P}^{i}-\mathbb{D}^{i}\right): \boldsymbol{\varepsilon}$.

The stress, obtained by derivation with respect to $\varepsilon$, and the actual elasticity tensor are:

$\boldsymbol{\sigma}=\sum_{i=1}^{I} \lambda^{i}\left(\mathbb{P}^{i}-\mathbb{D}^{i}\right): \boldsymbol{\varepsilon}$

$\mathbb{C}=\sum_{i=1}^{I} \lambda^{i}\left(\mathbb{P}^{i}-\mathbb{D}^{i}\right)$

These relations show that $\lambda^{i} \mathbb{D}^{i}$ can be regarded as the loss of stiffness of the mode $I$ of initial stiffness $\lambda^{i} \mathbb{P}^{i}$. This can be seen as an extension, in the 3D Kelvin framework, of the classical uni-dimensional approach of damage $\sigma=E(1-d) \varepsilon$ where $E$ is the Young modulus which corresponds in $1 \mathrm{D}$ to a Kelvin modulus and $d$ the scalar damage. The major hypothesis of the present model is that the tensors $\mathbb{D}^{i}$ remain internal into the original subspace $\mathcal{T}^{i}$ i.e.

$\mathbb{D}^{i}: \mathcal{T} \rightarrow \mathcal{T}^{i}$.

This hypothesis, already retained by Schreyer and Zuo (1995), allows the evolution of the symmetry group of the material if $\mathbb{D}^{i}$ acts on a subset of $\mathcal{T}^{i}$. It implies that the actual Kelvin decomposition of the material constitutes a partition of the initial one. In other words, the initial structure is never completely lost even if the material becomes fully anisotropic: this anisotropy will be related to the initial symmetry of the material. This point will be detailed in Section 5.

According to the framework of the GSM (generalized standard materials) (Halphen and Nguyen, 1975), the thermodynamic forces $\mathbb{Y}^{i}$ are associated with the damage variables $\mathbb{D}^{i}$ with respect to the free energy $\left(\mathbb{Y}^{i}=-\partial \rho \Psi / \partial \mathbb{D}^{i}\right)$. Considering the hypothesis of Eqs. (16) and (7), one obtains:

$2 \mathbb{\mho}^{i}=\lambda^{i} \boldsymbol{\varepsilon}^{i} \otimes \boldsymbol{\varepsilon}^{i}$

\subsection{Yield surface}

Following Biegler and Mehrabadi (1995), we shall consider in this introductory model that the free energy of each mode of the sound material is bounded by a maximum energy $W^{i}$. Thus $I \leqslant 6$ (the number of Kelvin modes of the sound material) material constants $W^{i}$ are required. These modal criterions (from Eq. 13) are:

$f^{i}\left(\boldsymbol{\varepsilon}, \mathbb{D}^{i}\right)=\frac{\lambda^{i}}{2} \boldsymbol{\varepsilon}:\left(\mathbb{P}^{i}-\mathbb{D}^{i}\right): \boldsymbol{\varepsilon}-W^{i} \leqslant 0$.

The damage evolution occurs if the criterion is reached $\left(f^{i}=0\right)$ and if the further evolution cannot be withstood with the actual damage level i.e. $f^{i}\left(\boldsymbol{\varepsilon}+\mathrm{d} \boldsymbol{\varepsilon}, \mathbb{D}^{i}\right)>0$ or equivalently

$\frac{\partial f^{i}}{\partial \boldsymbol{\varepsilon}}: \mathrm{d} \boldsymbol{\varepsilon}>0$.

In order to use the GSM framework, it is necessary to rewrite the criterion with respect to the thermodynamic force $\mathbb{Y}^{i}$. One obtains:

$f^{i}\left(\mathbb{Y}^{i}, \mathbb{D}^{i}\right)=\left\|\mathbb{Y}^{i}\right\|-\mathbb{Y}^{i}:: \mathbb{D}^{i}-W^{i}$,

where the symbol "::" represents the contraction over the nearest four indexes in the canonical basis or, equivalently, over the two indexes in the $\mathbf{B}^{i} \otimes \mathbf{B}^{j}$ basis.

\subsection{Evolution}

According to the GSM framework (in its associated form), the evolution of the state variables $\mathbb{D}^{i}$ obeys the normality rule which ensures the maximum dissipation principle:

$\mathrm{d} \mathbb{D}^{i}=\frac{\partial f^{i}}{\partial \bigvee^{i}} \mathrm{~d} \Lambda^{i}$,

where $\mathrm{d} \Lambda^{i}$ is a positive Lagrange multiplier. From Eqs. (20) and (17) one gets:

$\mathrm{d} \mathbb{D}^{i}=\left(\mathbf{K}^{i} \otimes \mathbf{K}^{i}-\mathbb{D}^{i}\right) \mathrm{d} \Lambda^{i}$.

$\mathbf{K}^{i}=\frac{\boldsymbol{\varepsilon}^{i}}{\left\|\boldsymbol{\varepsilon}^{i}\right\|}$. 
This key equation shows that the tensorial direction of the damage evolution depends upon both the actual damage state $\left(\right.$ via $\mathbb{D}^{i}$ ) and the actual strain (via $\mathbf{K}^{i}$ ). In case of non proportional straining, $\mathbf{K}^{i}$ changes, the damage tensor $\mathbb{D}^{i}$ does not remain colinear to itself thus the elastic tensor $\mathbb{C}$ (Eq. 15) and its symmetry group may change. Obtained from Eq. (18), the partial derivatives

$$
\begin{aligned}
& \frac{\partial f^{i}}{\partial \boldsymbol{\varepsilon}}=\lambda^{i}\left(\mathbb{P}^{i}-\mathbb{D}^{i}\right): \boldsymbol{\varepsilon} \\
& \frac{\partial f^{i}}{\partial \mathbb{D}^{i}}=-\frac{\lambda^{i}}{2} \boldsymbol{\varepsilon}^{i} \otimes \boldsymbol{\varepsilon}^{i}
\end{aligned}
$$

allow one (using Eq. 16 from which $\mathrm{d} \mathbb{D}^{i}: \boldsymbol{\varepsilon}=\mathrm{d} \mathbb{D}^{i}: \boldsymbol{\varepsilon}^{i}$ ) to express the consistency equation $\mathrm{d} f^{i}\left(\boldsymbol{\varepsilon}, \mathbb{D}^{i}\right)=0$ as follows:

$2 \boldsymbol{\varepsilon}:\left(\mathbb{P}^{i}-\mathbb{D}^{i}\right): \mathrm{d} \boldsymbol{\varepsilon}=\boldsymbol{\varepsilon}: \mathrm{d} \mathbb{D}^{i}: \boldsymbol{\varepsilon}$.

Together with Eq. (22), this gives the value of the Lagrange multiplier:

$\mathrm{d} \Lambda^{i}=2 \frac{\boldsymbol{\varepsilon}:\left(\mathbb{P}^{i}-\mathbb{D}^{i}\right): \mathrm{d} \boldsymbol{\varepsilon}}{\boldsymbol{\varepsilon}:\left(\mathbb{P}^{i}-\mathbb{D}^{i}\right): \boldsymbol{\varepsilon}}$.

The intrinsic dissipation evolution is given by the product of thermodynamic forces and fluxes (Halphen and Nguyen, 1975) i.e., for the mode $I$ :

$\mathrm{d} \mathcal{D}^{i}=\mathbb{Y}^{i}:: \mathrm{d} \mathbb{D}^{i}$

This can be expressed, using successively Eqs. (17), (26) and (24), as:

$$
\begin{aligned}
& \mathrm{d} \mathcal{D}^{i}=\frac{\lambda^{i}}{2} \boldsymbol{\varepsilon}: \mathrm{d} \mathbb{D}^{i}: \boldsymbol{\varepsilon}, \\
& =\lambda^{i} \boldsymbol{\varepsilon}:\left(\mathbb{P}^{i}-\mathbb{D}^{i}\right): \mathrm{d} \boldsymbol{\varepsilon}, \\
& =\frac{\partial f^{i}}{\partial \boldsymbol{\varepsilon}}: \mathrm{d} \boldsymbol{\varepsilon} .
\end{aligned}
$$

The last expression, together with 19, leads to:

$\mathrm{d} \mathcal{D}^{i}>0$

in case of damage evolution. Thus, the global dissipation $\mathrm{d} \mathcal{D}=\sum_{i=1}^{I} \mathrm{~d} \mathcal{D}^{i}$ is positive as required by the second principle of the thermodynamics. From Eqs. (30) and (27) one obtains:

$$
\begin{aligned}
& \mathrm{d} \mathcal{D}^{i}=\frac{\lambda^{i}}{2} \boldsymbol{\varepsilon}:\left(\mathbb{P}^{i}-\mathbb{D}^{i}\right): \boldsymbol{\varepsilon} \mathrm{d} \Lambda^{i}, \\
& =\rho \Psi^{i} \mathrm{~d} \Lambda^{i}, \\
& =W^{i} \mathrm{~d} \Lambda^{i},
\end{aligned}
$$

where $\rho \Psi^{i}$ denote the free energy stored in the mode $i$, consistently with Eq. (14). From above $\boldsymbol{\varepsilon}:\left(\mathbb{P}^{i}-\mathbb{D}^{i}\right): \boldsymbol{\varepsilon}=$ $\boldsymbol{\varepsilon}^{i}:\left(\mathbb{P}^{i}-\mathbb{D}^{i}\right): \boldsymbol{\varepsilon}^{i}=2 W^{i} / \lambda^{i}>0$ for any strain $\boldsymbol{\varepsilon}$ which belongs to the (ellipsoidal) yield surface $f^{i}=0$. A generic strain $\boldsymbol{\varepsilon}^{*}$ can be expressed as $\boldsymbol{\varepsilon}^{*}=\boldsymbol{\varepsilon}^{* \perp}+\kappa \boldsymbol{\varepsilon}^{i}$ where $\boldsymbol{\varepsilon}^{* \perp}$ belongs to the space $\mathcal{T}-\mathcal{T}^{i}$. Thus $\boldsymbol{\varepsilon}^{*}:\left(\mathbb{P}^{i}-\mathbb{D}^{i}\right): \boldsymbol{\varepsilon}^{*}=$ $2 \kappa^{2} W^{i} / \lambda^{i}>0$ and this shows that $\mathbb{P}^{i}-\mathbb{D}^{i}$ and $\mathbb{C}$ are positive semidefinite.

\subsection{Algorithm}

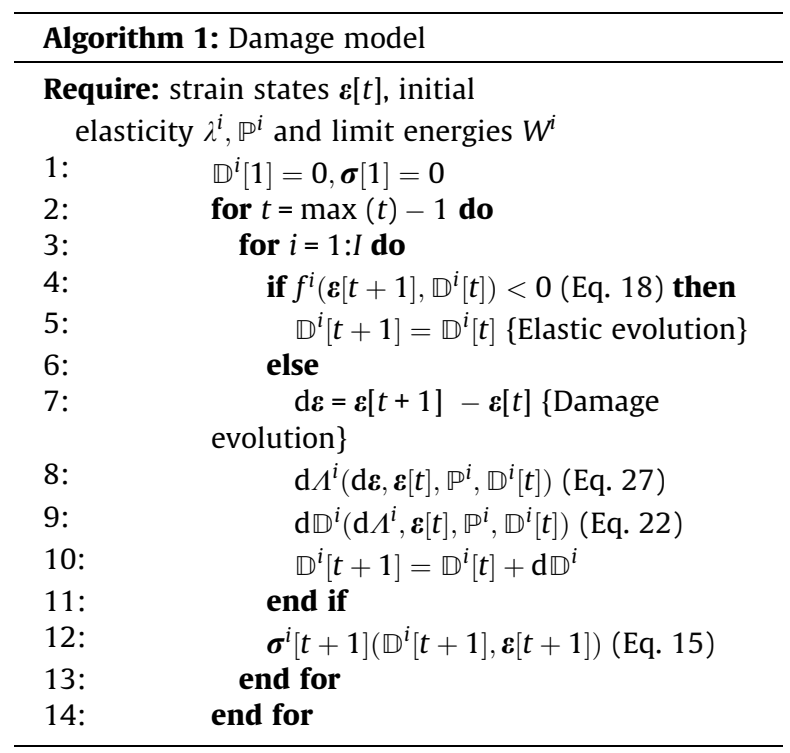

The proposed model has been implemented in a simple integration scheme as shown by Algorithm (1). The index $t$ refers here to the actual step of the calculus. Using tensorial basis (Eq. 1), the $2 \times I$ fourth-rank tensors $\mathbb{P}^{i}$ and $\mathbb{D}^{i}$ are stored in $6 \times 6$ matrices and the second-rank tensors $\boldsymbol{\sigma}, \boldsymbol{\varepsilon}$ in $6 \times 1$ vectors. The algorithm was programmed within the Matlab software and the main body of the program is less than ten lines. It concerns a strain-driven path which can be used as a UMAT in the ABAQUS software. The robustness test of Matallah and La Borderie (2009), which consists in a strain path of the form $\varepsilon=\cos (\theta) \sin (\theta)$ $2 n) \mathbf{B}^{1}+\sin (\theta) \sin (\theta / 2 n) \mathbf{B}^{2}+(1-\cos (\theta / 2 n)) \mathbf{B}^{3}$ where $\theta$ ranges from 0 to $2 \pi n$, (for many $n$ ) has been successfully tested (not presented).

\subsection{Example of the initially isotropic material and two proportional loadings}

For sake of simplicity this example refers to an initially isotropic material (Eq. 11). In Table 1, the Kelvin moduli $(3 K, 2 \mu)$ correspond to a Young modulus $E=1$ and a Poisson's ratio $v=1 / 3, W^{H}$ and $W^{D}$ are the limit energies of respectively the hydrostatic $(\mathrm{H})$ and deviatoric (D) modes (Eq. 18).

The first loading path is an oedometric tension defined as follows:

$\boldsymbol{\varepsilon}^{1}=\varepsilon(t) \mathbf{B}^{1}$,

where $\varepsilon(t)$ evolves from 0 to 1 then back to 0 and $t$ represents time. From Eq. (23), the normed tensors $\mathbf{K}^{i}$ are $\mathbf{K}^{H}$ and

Table 1

Material constants.

\begin{tabular}{llll}
\hline $3 K$ & $2 \mu$ & $W^{\mathrm{H}}$ & $W^{\mathrm{D}}$ \\
\hline 3 & $3 / 4$ & $1 / 4$ & $1 / 6$ \\
\hline
\end{tabular}


$\mathbf{K}^{D 1}$ whose respective components are $(1,1,1,0,0,0) / \sqrt{3}$ and $(2,-1,-1,0,0,0) / \sqrt{6}$ in the basis $\mathbf{B}^{i}$. At the beginning of yielding, both the damage tensors $\mathbb{D}^{H}$ and $\mathbb{D}^{D}$ are null. Eq. (22) shows that, in such case of proportional loading, the damage tensor $\mathbb{D}^{H}$ remains colinear to $\mathbf{K}^{H} \otimes \mathbf{K}^{H}$ and $\mathbb{D}^{D}$ colinear to $\mathbf{K}^{D 1} \otimes \mathbf{K}^{D 1}$. Thus one can write $\mathbb{D}^{H}=d^{H} \mathbf{K}^{H} \otimes \mathbf{K}^{H}=\mathbb{P}^{H}, \mathbb{D}^{D}=d^{D 1} \mathbf{K}^{D 1} \otimes \mathbf{K}^{D 1}$ and, from Eq. (15), the actual Hooke law is:

$\mathbb{C}=3 K\left(1-d^{H}\right) \mathbb{P}^{H}+2 \mu\left(\mathbb{P}^{D}-d^{D 1} \mathbf{K}^{D 1} \otimes \mathbf{K}^{D 1}\right)$.

This expression shows that the response of the material to an hydrostatic strain is reduced but still isotropic and that the response to a deviatoric strain is no more isotropic: the 4-dimensional subspace of projector $\mathbb{P}^{D}-\mathbf{K}^{D 1} \otimes \mathbf{K}^{D 1}$ corresponds to an unchanged Kelvin modulus $2 \mu$ but the 1dimensional subspace of projector $\mathbf{K}^{D 1} \otimes \mathbf{K}^{D 1}$ corresponds to a reduced Kelvin modulus $2 \mu\left(1-d^{D 1}\right)$. The consistency Eq. (27) simplifies in this case to $\mathrm{d} \Lambda^{H}=\mathrm{d} \Lambda^{D}=2 \mathrm{~d} \varepsilon / \varepsilon$. This allows one to obtain, from Eq. $(22): d^{H}=1-\left(\varepsilon_{y}^{H} / \varepsilon\right)^{2}$ and $d^{D 1}=1-\left(\varepsilon_{y}^{D} / \varepsilon\right)^{2}$ where $\varepsilon_{y}^{H}=\sqrt{2 W^{H} / K}$ and $\varepsilon_{y}^{D}=\sqrt{3 W^{D} / 2 \mu}$ are the strains at which yielding begins, given by their respective initial yield conditions (Eq. 18). These strains correspond respectively to the points $A$ and $A^{\prime}$ on Fig. 1. Between points 0 and $\mathrm{A} f^{H}<0$ and $f^{D}<0$, the behavior is linear elastic of slope $K+4 \mu / 3$ in the coordinates of the Fig. 1. From $A$ to $A^{\prime} f^{H}=0$ and $f^{D}<0, d^{H}$ evolves alone. From $\mathrm{A}^{\prime}$ to $\mathrm{B}, f^{H}=0$ and $f^{D}=0, d^{H}$ and $d^{D}$ increase. In both cases, one can easily verify from above that the stress to strain curve in Fig. 1 has an hyperbolic shape, consistently with the criterion of constant modal energy. The unloading BO shows a reduced linear elasticity.

The strain path is continued by an imposed shearing

$\boldsymbol{\varepsilon}^{2}=\varepsilon(t) \mathbf{B}^{4}$,

where $\varepsilon$ evolves from 0 to 1 then back to 0 . From Eq. (23) one obtains $\mathbf{K}^{\mathrm{D2}}=\mathbf{B}^{4}$. Eq. (22) shows that $\mathbb{D}^{D}$ now evolves from part along $\mathbf{K}^{D 2} \otimes \mathbf{K}^{D 2}$ and from part along $\mathbf{K}^{D 1} \otimes \mathbf{K}^{D 1}$ (which is already contained in $\mathbb{D}^{D}$ from previous loading). The actual elasticity law can be written as:

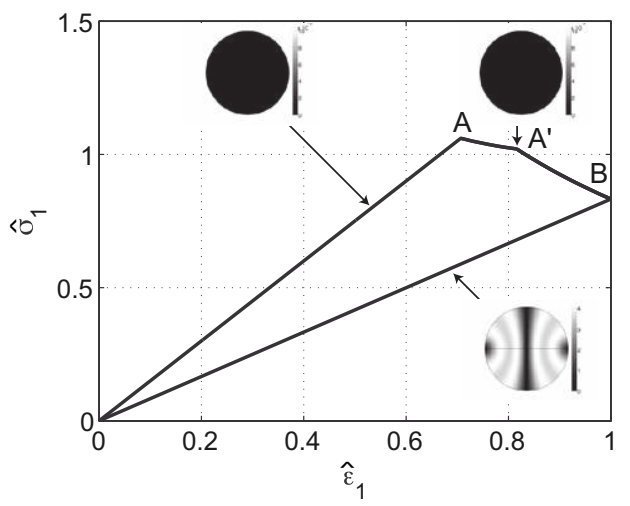

Fig. 1. First loading path: oedometric tension and pole figures of the elasticity tensor.

$$
\begin{aligned}
\mathbb{C}= & 3 K\left(1-d^{H}\right) \mathbb{P}^{H}+2 \mu\left(\mathbb{P}^{D}-d^{D 1} \mathbf{K}^{D 1} \otimes \mathbf{K}^{D 1}\right. \\
& \left.-d^{D 2} \mathbf{K}^{D 2} \otimes \mathbf{K}^{D 2}\right) .
\end{aligned}
$$

The evolution of $\left(d^{H}, d^{D 1}, d^{D 2}\right)$ will be detailed in Section 4.3. However above results still show that $d^{H}$ is not concerned by the present evolution (its value remains constant). The evolution CC'D (Fig. 2), which involves damage, exhibit similar hyperbolic shape as for the previous loading.

Eqs. (37) and (39) correspond to a Kelvin decomposition of the material actual elasticity. Easily obtained in these cases of proportional loading, such result is generalized in Section 4.

Fig. 3 shows the strain path and the deviatoric yield surfaces $f^{D}=0$ at points $0, \mathrm{~B}$ (end of tension) and $\mathrm{D}$ (end of shear) in both the coordinates $\left(\mathbf{B}^{1}, \mathbf{B}^{4}\right)$ and $\left(\mathbf{K}^{D 1}, \mathbf{K}^{D 2}\right)$. From the above expressions of $\mathbb{D}^{D 1}$ and $\mathbb{D}^{D 2}, f^{D}=0$ can be written as $\mu \boldsymbol{\varepsilon}:\left(\mathbb{P}^{D}-d^{D 1} \mathbf{K}^{D 1} \otimes \mathbf{K}^{D 1}-d^{D 2} \mathbf{K}^{D 2} \otimes \mathbf{K}^{D 2}\right): \boldsymbol{\varepsilon}-W^{D}=0$. For a strain such as $\boldsymbol{\varepsilon}=x \mathbf{K}^{D 1}+y \mathbf{K}^{D 2}$, this leads to $x^{2}\left(1-d^{D 1}\right)+y^{2}\left(1-d^{D 2}\right)=W^{D} / \mu$. This shows that the yield surfaces in the coordinates $\left(\mathbf{K}^{D 1}, \mathbf{K}^{D 2}\right)$ are elliptic and, in particular, circular for the undamaged material.

\section{Kelvin's analysis of the model}

\subsection{Second-rank tensor basis of the damage tensors}

At this stage, the model is fully defined, however, the mechanical significance of the fourth-rank damage tensors $\mathbb{D}^{i}$ is still not unveiled. By construction (Eq. 22), they are fourth-rank tensors whose matrix of component in the basis $\mathbf{B}^{i} \otimes \mathbf{B}^{j}$ is $6 \times 6$ is symmetric. Thus they accept a diagonal form, i.e. a Kelvin decomposition in which, as the image of $\mathbb{D}^{i}$ is the subset $\mathcal{T}^{i} \subset \mathcal{T}$ (from Eq. 16), many eigenvalues can be null. This decomposition is written as follows:

$$
\begin{aligned}
& \mathbb{D}^{i}=\sum_{p=1}^{p^{i}} d^{i p} \mathbb{P}^{i p}, \\
& \mathbb{P}^{i p}=\sum_{m=1}^{M^{i p}} \mathbf{E}^{i p m} \otimes \mathbf{E}^{i p m},
\end{aligned}
$$

where $d^{i p}$ are the eigenvalues, the $\mathbf{E}^{i p m}$ their $M^{i p}$ corresponding eigenvectors and $\mathbb{P}^{i p}$ the associated fourth-rank

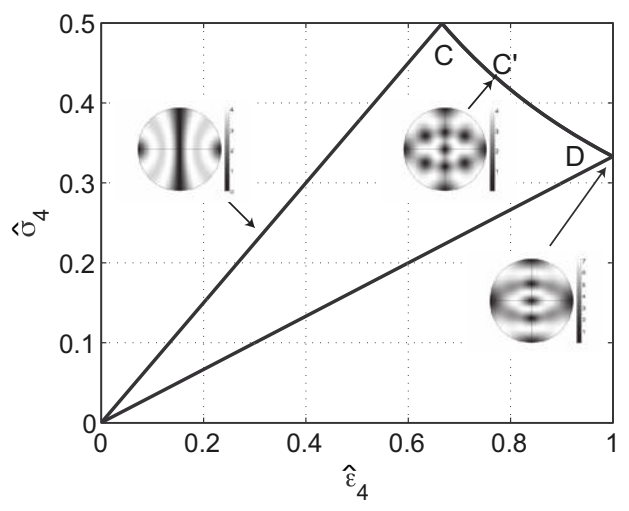

Fig. 2. Second loading path: shear and pole figures of the elasticity tensor. 

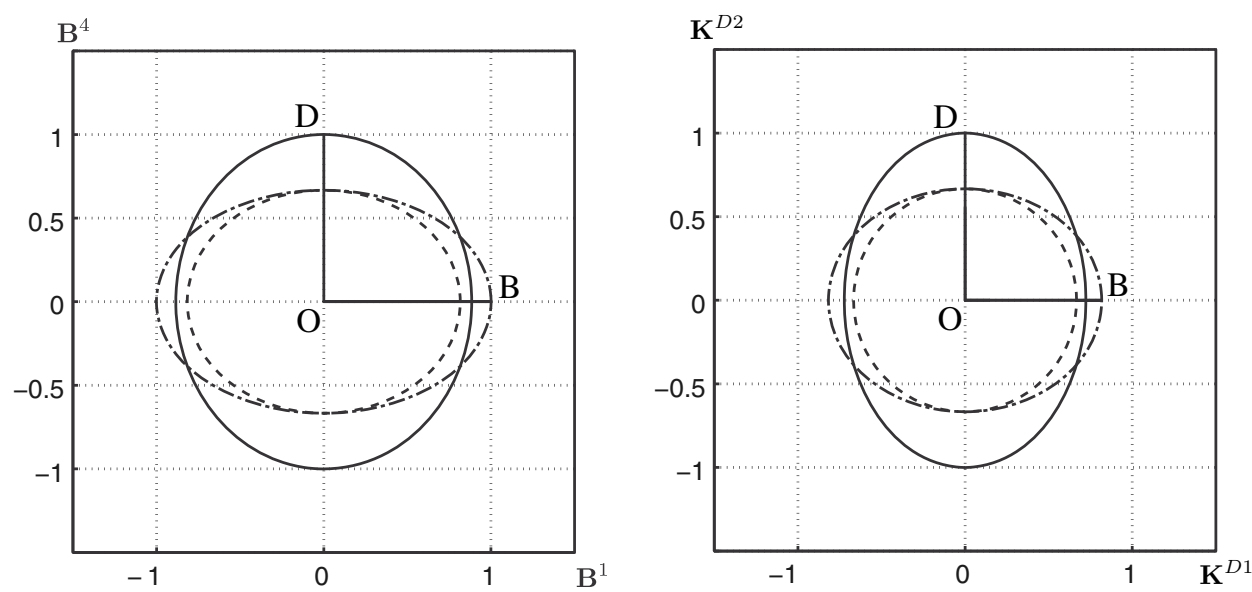

Fig. 3. Strain path and deviatoric yield surfaces in the strain space: initial (dashed), at point B (dash dotted), and D (plain).

projectors onto subspaces $\mathcal{T}^{i p}$ of basis $\mathbf{E}^{i p m}$ where $(i, p)$ are fixed. From the normality (Eq. 22) and because $\mathbf{K}^{i} \in \mathcal{T}^{i}$ (Eqs. 7, 23), $\mathbb{D}^{i}$ is internal in $\mathcal{T}^{i}$ as envisaged in Eq. (16) and the set of $\mathbf{E}^{i p m}$, where $i$ is fixed alone, is an orthonormal basis of $\mathcal{T}^{i}$ (a particular choice of the generic basis $\mathbf{E}^{\text {in }}$ defined as in Eq. 2). The set of all the $\mathbf{E}^{i p m}$ is an orthonormal basis of $\mathcal{T}$. This leads to:

$\mathcal{T}^{i}=\bigoplus_{p=1}^{P^{i}} \mathcal{T}^{i p}, \quad \mathbb{P}^{i}=\sum_{p=1}^{P^{i}} \mathbb{P}^{i p}, \quad \sum_{p=1}^{P^{i}} M^{i p}=N^{i}$.

Table 2 helps the reader for these notations. This Kelvin decomposition of $\mathbb{D}^{i}$ consitutes a partition of the Kelvin decomposition for the sound material (Eq. 2) and is consistent with it for $d^{i p}=0$. Above results and the Hooke law (Eq. 15) show that the Kelvin decomposition of $\mathbb{C}$ shares the same projectors as $\mathbb{D}^{i}$ :

$\mathbb{C}=\sum_{i=1}^{I} \lambda^{i} \sum_{p=1}^{P^{i}}\left(1-d^{i p}\right) \mathbb{P}^{i p}$.

In the manner of Eqs. (6) and (7), we have:

$\boldsymbol{\sigma}^{i p}=\lambda^{i}\left(1-d^{i p}\right) \boldsymbol{\varepsilon}^{i p}$

$\boldsymbol{\sigma}^{i p}=\mathbb{P}^{i p}: \boldsymbol{\sigma}, \quad \boldsymbol{\varepsilon}^{i p}=\mathbb{P}^{i p}: \boldsymbol{\varepsilon}$,

$\boldsymbol{\sigma}=\sum_{i=1}^{I} \sum_{p=1}^{P^{i}} \boldsymbol{\sigma}^{i p}, \quad \boldsymbol{\varepsilon}=\sum_{i=1}^{I} \sum_{p=1}^{P^{i}} \boldsymbol{\varepsilon}^{i p}$.

Table 2

The index system.

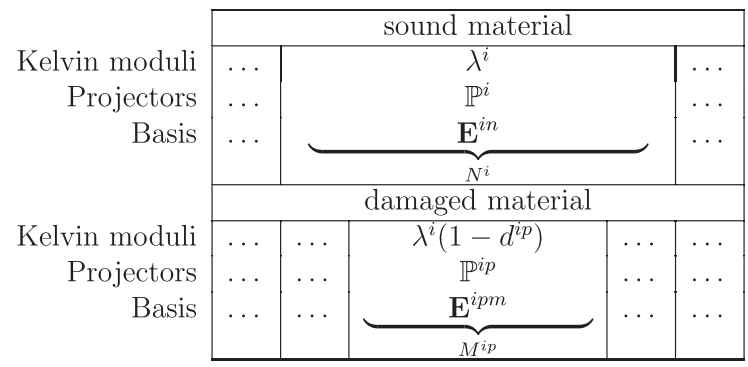

The free energy Eq. (13) and the yield criterions Eq. (18) can be written as:

$2 \rho \Psi=\sum_{i=1}^{I} \lambda^{i} \sum_{p=1}^{P^{i}}\left(1-d^{i p}\right) \boldsymbol{\varepsilon}^{i p}: \boldsymbol{\varepsilon}^{i p}$,

$f^{i}=\frac{\lambda^{i}}{2} \sum_{p=1}^{p^{i}}\left(1-d^{i p}\right) \boldsymbol{\varepsilon}^{i p}: \boldsymbol{\varepsilon}^{i p}-W^{i}$.

This shows that the criterion $f^{i}=0$ is (hyper) elliptic, centered on tensor $\mathbf{0}$ and convex in any cases. Expressions Eq. (43), (44) and (47) clearly indicate that the $d^{i p}$ represent a loss of stiffness with respect to the sound state of the material as defined for damage mechanics. The projectors $\mathbb{P}^{i p}$ define the tensorial subset of influence of each corresponding scalar damage $d^{i p}$ whose maximum number is 6. A given mode $i$ can "vanish" if the corresponding $d^{i p} \rightarrow 1, \forall p$. If this is true for all modes, $d^{i p} \rightarrow 1, \forall(i, p)$, the material is fully damaged and associates an infinitively small stress to any strain.

\subsection{Scalar damage evolution}

The calculation of $d^{i p}$ and $\mathbf{E}^{i p}$ requires the diagonalization of $\mathbb{D}^{i}$. Although easy in the basis $\mathbf{B}^{i}$, this operation is not necessary for the model operation. The positiveness of the dissipation, in its expression of Eq. (29), can be transformed by using successively the normality Eq. (22), the expression of $\mathbb{D}^{i}$ in Eq. (40) and the orthogonal projector relation in Eq. (42):

$$
\begin{gathered}
\boldsymbol{\varepsilon}:\left(\mathbf{K}^{i} \otimes \mathbf{K}^{i}-\mathbb{D}^{i}\right): \boldsymbol{\varepsilon}>0 \\
\boldsymbol{\varepsilon}^{i}: \boldsymbol{\varepsilon}^{i}-\sum_{p=1}^{P^{i}} d^{i p} \boldsymbol{\varepsilon}^{i p}: \boldsymbol{\varepsilon}^{i p}>0 \\
\sum_{p=1}^{p^{i}}\left(1-d^{i p}\right) \boldsymbol{\varepsilon}^{i p}: \boldsymbol{\varepsilon}^{i p}>0
\end{gathered}
$$

Recalling that this equation applies for any choice of $\varepsilon$ (such as $f^{i}=0$ ) yields $d^{i p}<1$. As a consequence $\mathbb{C}$ (Eq. 43) has positive Kelvin moduli thus is positive definite and the free energy (Eq. 47) is positive. The damage evolution 


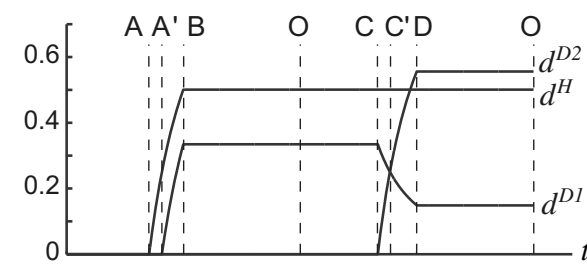

Fig. 4. Scalar damage levels v.s. fictitious time.

$\mathrm{d} \mathbb{D}^{i}$ (Eq. 22) is expressed in the basis $\mathbf{E}^{i p m}$ as the difference between the symmetric matrix of $\mathbf{K}^{i} \otimes \mathbf{K}^{i}$ and the diagonal one of $\mathbb{D}^{i}$. A diagonal term of $\mathrm{d} \mathbb{D}^{i}$ has the form $k^{2}-d^{i p}$ where $k=\mathbf{K}^{i}: \mathbf{E}^{i p m}$ is a component of the normed tensor $\mathbf{K}^{i}$ thus $0 \leqslant k^{2} \leqslant 1$. This term can be negative, leading to a reduction of $d^{i p}$ and this recovery capability differs from classical damage models. The highest rate of damage reduction is given when $k=0$ and this gives, from Eq. (22), $\mathrm{d} d^{i p}=-d^{i p} \mathrm{~d} \Lambda^{i}$ thus, as $\mathrm{d} \Lambda^{i}>0, d^{i p}$ cannot be negative.

$0 \leqslant d^{i p}<1$.

This case corresponds to an evolution of damage in a tensorial direction $\mathbf{K}^{i}$ orthogonal to the direction $\mathbf{E}^{i p m}$ related to the considered damage $d^{i p}$. Such case is met in Section 4.3.

\subsection{Example}

In this part we apply these results to the example of Section 3.5. As envisaged, the hydrostatic damage intensity $d^{H}$ increases continuously during $\mathrm{AB}$ but remains constant during path $C D$ in which the strain $\varepsilon^{2}$ is purely deviatoric. During $A^{\prime} B, d^{D 1}$ increases (see Fig. 4), the material becomes weaker with respect to the direction $\mathbf{K}^{D 1}$ which is the deviatoric part of the actually imposed strain $\boldsymbol{\varepsilon}^{1}$. From Eq. (37), $\mathbf{K}^{D 1}$ is an eigenvector of $\mathbb{D}^{D}$ i.e. $\mathbf{K}^{D 1}=\mathbf{E}^{D 1}$ (this simplicity is due to the proportionality of the loading). During $C D, d^{D 2}$ increases, concerning the direction $\mathbf{K}^{D 2}=\mathbf{E}^{D 2}$ of the imposed strain $\varepsilon^{2}$. In the same time $\mathrm{d}^{D 1}$ decreases as envisaged in Section 3.5. This can be observed on Fig. 3: the yield surface radius along $\mathbf{K}^{D 1}$ decreases from state B to state $\mathrm{D}$. The rate of increase of $d^{D 2}$ is compared to the rate of decrease of $d^{D 1}$ at point $C$ (where $d^{D 2}=0$ ). In the basis $\left(\mathbf{K}^{D 1}, \mathbf{K}^{D 2}\right)$ (other dimensions are not concerned), one can write the damage evolution $\mathrm{d} \mathbb{D}^{D}$ (Eq. 22) as follows:

$$
\left[\begin{array}{cc}
\mathrm{d} d^{D 1} & 0 \\
0 & \mathrm{~d} d^{D 2}
\end{array}\right]=\left[\begin{array}{cc}
-d^{D 1} & 0 \\
0 & 1
\end{array}\right] \mathrm{d} \Lambda^{D} .
$$

According to this expression, $\mathrm{d} d^{D 1}=-d^{D 1} \mathrm{~d} d^{D 2}$ thus the rate of decrease of the inactive damage $d^{D 1}$ is lower than the rate of increase of active damage $d^{D 2}$. This case corresponds to the case of fastest damage decrease $(k=0)$ envisaged in Section 4.2. The dissipation (Eq. 28) can be expressed as $\mathrm{d} \mathcal{D}^{D}=2 \mu \varepsilon \mathrm{d} \varepsilon>0$ which does not involve the value of the inactive damage $d^{D 1}$.

\section{Symmetry analysis and Curie principle}

\subsection{Symmetry group of the actual elasticity tensor}

From Theorem 10, the symmetry group of the elasticity tensor is the intersection of the symmetry groups of its projectors. These ones have the structure of an elasticity tensor (with $6-M^{i p}$ null eigenvalues) then their symmetry group belongs to one of the eight possible between isotropic, cubic, transverse isotropic, tetragonal, orthotropic, trigonal, monoclinic and tricilinic) (Huo and Del Piero, 1991; Forte and Vianello, 1996). From theorem 3, the symmetry group of a projector is the set of orthogonal transformations internal in the corresponding subspace. For the sound material, the symmetry group of the stiffness tensor (Eq. 5) is:

$$
\begin{aligned}
\mathcal{S}\left(\mathbb{C}, \mathbb{D}^{i}\right. & =0)=\bigcap_{i=1}^{I} \mathcal{S}\left(\mathbb{P}^{i}\right), \\
& =\bigcap_{i=1}^{I}\left\{Q \in \mathcal{S O}(3) / Q\left(\mathbf{E}^{i n} \in \mathcal{T}^{i}\right) \in \mathcal{T}^{i}\right\} .
\end{aligned}
$$

Similarly, for the damaged material (Eq. 43) the symmetry group is:

$$
\begin{aligned}
\mathcal{S}(\mathbb{C}) & =\bigcap_{i=1}^{I} \bigcap_{p=1}^{p^{i}} \mathcal{S}\left(\mathbb{P}^{i p}\right), \\
& =\bigcap_{i=1}^{I} \bigcap_{p=1}^{p^{i}}\left\{Q \in \mathcal{S O}(3) / Q\left(\mathbf{E}^{i p m} \in \mathcal{T}^{i p}\right) \in \mathcal{T}^{i p}\right\} .
\end{aligned}
$$

As soon as the $\mathbf{E}^{i p m}$ constitute a particular choice of the $\mathbf{E}^{\text {in }}$, it is easy to verify that the first group contains the second i.e. that the damaged material is less (or equally) symmetric than the sound one.

\subsection{Symmetry group of the causes}

The causes have two origins: the material's actual state defined by the actual stiffness tensor $\mathbb{C}$ and the mechanical solicitation. This last one is represented by both the stress $\boldsymbol{\sigma}$ and the strain $\boldsymbol{\varepsilon}$ which have, in general, a different symmetry group as soon as they are not proportional tensors. However, the Kelvin decomposition decomposes them into proportional parts (Eq. 6 for the sound material and Eq. (44) for the damaged one). The symmetry group of $\boldsymbol{\sigma}^{i p}$ and $\boldsymbol{\varepsilon}^{i p}$ are the same thus are capable to describe the symmetry group of the mechanical solicitation. Under this assumption, the symmetry group of the causes is

$\mathcal{S}$ (causes) $=\mathcal{S}(\mathbb{C}) \bigcap_{i=1}^{I} \bigcap_{p=1}^{p^{i}} \mathcal{S}\left(\boldsymbol{\varepsilon}^{i p}\right)$.

\subsection{Symmetry group of the consequences}

At each infinitesimal step of the damage evolution the stiffness of the material evolves towards $\mathbb{C}+\mathrm{d} \mathbb{C}$. This term is considered as the consequence of the process. The normality (Eq. 22) and the expression of $\mathbb{D}^{i}$ (Eq. 40) yields:

$\mathcal{S}\left(\mathrm{d} \mathbb{D}^{i}\right)=\bigcap_{p=1}^{p^{i}} \mathcal{S}\left(\mathbb{P}^{i p}\right) \bigcap \mathcal{S}\left(\mathbf{K}^{i} \otimes \mathbf{K}^{i}\right)$.

From above, the stiffness tensor expression (Eq. 15) and its symmetry group (Eq. 53), one deduces the symmetry group of the consequences: 


$$
\begin{aligned}
& \mathrm{d} \mathbb{C}=-\sum_{i=1}^{I} \lambda^{i} \mathrm{~d} \mathbb{D}^{i}, \\
& \mathcal{S}(\mathrm{d} \mathbb{C})=\bigcap_{i=1}^{I} \mathcal{S}\left(\mathrm{d} \mathbb{D}^{i}\right), \\
& \mathcal{S}(\mathbb{C}+\mathrm{d} \mathbb{C})=\mathcal{S}(\mathbb{C}) \bigcap_{i=1}^{I} \mathcal{S}\left(\mathbf{K}^{i} \otimes \mathbf{K}^{i}\right),
\end{aligned}
$$

\subsection{Verification of the Curie principle}

The Corollary 1 and the definition of $\mathbf{K}^{i}$ (Eq. 23) lead to:

$\mathcal{S}\left(\boldsymbol{\varepsilon}^{i}\right)=\mathcal{S}\left(\mathbf{K}^{i}\right) \subset \mathcal{S}\left(\mathbf{K}^{i} \otimes \mathbf{K}^{i}\right)$.

From the partition of the projectors (Eq. 42), $\boldsymbol{\varepsilon}^{i}=\sum_{p=1}^{p^{i}} \boldsymbol{\varepsilon}^{i p}$ thus:

$$
\begin{aligned}
& Q \in \bigcap_{p=1}^{P^{i}} \mathcal{S}\left(\boldsymbol{\varepsilon}^{i p}\right) \Rightarrow Q \in \mathcal{S}\left(\boldsymbol{\varepsilon}^{i}\right), \\
& \Longleftrightarrow \bigcap_{p=1}^{P^{i}} \mathcal{S}\left(\boldsymbol{\varepsilon}^{i p}\right) \subset \mathcal{S}\left(\boldsymbol{\varepsilon}^{i}\right) .
\end{aligned}
$$

From the inclusions relations (Eqs. 59 and 60) one deduces the final relation:

$$
\begin{aligned}
& \bigcap_{p=1}^{p^{i}} \mathcal{S}\left(\boldsymbol{\varepsilon}^{i p}\right) \subset \mathcal{S}\left(\mathbf{K}^{i} \otimes \mathbf{K}^{i}\right), \\
& \mathcal{S}(\mathbb{C}) \bigcap_{i=1}^{I} \bigcap_{p=1}^{P^{i}} \mathcal{S}\left(\boldsymbol{\varepsilon}^{i p}\right) \subset \mathcal{S}(\mathbb{C}) \bigcap_{i=1}^{I} \mathcal{S}\left(\mathbf{K}^{i} \otimes \mathbf{K}^{i}\right),
\end{aligned}
$$

which corresponds to the verification of the Curie principle, i.e. the symmetry group of the consequences (the right hand term, Eq. 58) includes the one of the causes (the left hand term, Eq. 54). The consequences are at least as symmetrical as the causes, as envisaged by the Curie principle. One can remark that the first inclusion relation (Eq. 59 ) is simply due to the use of fourth-rank projectors. On the contrary, the second one (Eq. 60) is due to the expression of $\mathrm{d} \mathbb{D}^{i}$ (Eq. 22) as its first member depends upon $\mathbf{K}^{i}=\mathbb{P}^{i}: \boldsymbol{\varepsilon} /\left\|\mathbb{P}^{i}: \boldsymbol{\varepsilon}\right\|$ where $\mathbb{P}^{i}$ is the projector associated with the sound state (and not the actual ones $\mathbb{P}^{i p}$ ). This observation can be useful for further evolution of the model.

\subsection{Example and remarks}

The symmetry group of the stiffness tensor evolves all along the damage process presented in the example (Sections 3.5 and 4.3). In order to have a visual interpretation of the symmetry group of the stiffness tensor $\mathbb{C}$, pole figures (François et al., 1998; Diner et al., 2011) are used. They consist in a measurement of a distance $\xi(\mathbb{C}, \mathbf{n})$ between $\mathbb{C}$ and its symmetric $S_{\mathbf{n}}(\mathbb{C})$ with respect to all planes whose normals $\mathbf{n}$ range the half unit sphere:

$\xi(\mathbb{C}, \mathbf{n})=\frac{\left\|\mathbb{C}-S_{\mathbf{n}}(\mathbb{C})\right\|}{\|\mathbb{C}\|}$, where $\|\mathbb{C}\|=\sqrt{C_{\mathrm{pqrs}} C_{\mathrm{pqrs}}}$. The result is presented on grayscale pole figures (thumbnails in Figs. 1, 2 and 6). Black corresponds to $\xi=0$, denoting the existence of a symmetry plane of normal $\mathbf{n}$. White corresponds to a large discrepancy between $\mathbb{C}$ and $S_{\mathbf{n}}(\mathbb{C})$. A fully black pole figure denotes the isotropy. The eight possible symmetry groups for the elasticity tensors can be distinguished by the number (and orientation) of the dark spots (see François et al., 1998 for details).

Concerning the example of Section 3.5, the symmetry group of the hydrostatic damage tensor $\mathbb{D}^{H}=d^{H} \mathbb{P}^{H}$ remains $\mathcal{S O}(3)$ and thus does not affect the symmetry group of the material since, $\mathcal{S} \cap \mathcal{S O}(3)=\mathcal{S}$ for all symmetry group $\mathcal{S}$ ). From $A^{\prime}$ to B the deviatoric damage tensor $\mathbb{D}^{D}$ evolves proportionally to $\mathbf{E}^{D 1} \otimes \mathbf{E}^{D 1}$. The symmetry group of $\mathbf{E}^{D 1}=\mathbf{K}^{D 1}$ is obviously transverse isotropic (of axis $\mathbf{e}^{1}$ ), thus $\mathbf{E}^{D 1} \otimes \mathbf{E}^{D 1}$ (from Corollary 1) and $\mathbb{C}$ (from theorem 10) are also transverse isotropic, as shown by the pole figure at $\mathrm{C}$ in Fig. 1. From $C$ to $D, \mathbb{D}^{D}$ evolves also along the projector $\mathbf{E}^{D 2} \otimes \mathbf{E}^{D 2}$. However, if $\mathbf{E}^{D 2}=\mathbf{K}^{D 2}=\mathbf{B}^{4}$ is orthotropic (the symmetry group is $D_{2}$ ), from Corollary $1 \mathbf{E}^{D 2} \otimes \mathbf{E}^{D 2}$ is tetragonal because the elements of $D_{4}$ transform $\mathbf{B}^{4}$ in $\pm \mathbf{B}^{4}$ (for example the rotation $\mathcal{R}_{\left[\mathbf{e}^{1}, \pi / 2\right]}\left(\mathbf{B}^{4}\right)=-\mathbf{B}^{4}$ ). Again from theorem 10 , the symmetry groups of $\mathbb{D}$ and $\mathbb{C}$ are the intersection of the transverse isotropy of $\mathbf{E}^{D 1} \otimes \mathbf{E}^{D 1}$ and this tetragonal symmetry. Sharing the same axis $\left(\mathbf{e}^{1}, \mathbf{e}^{2}, \mathbf{e}^{3}\right)$, the transverse isotropy includes the tetragonal symmetry thus $\mathbb{D}$ and $\mathbb{C}$ are tetragonal as shown in Fig. 2 which shows the five spots (normal of symmetry planes) characteristic of it. Point C' is the point where $d^{D 1}=d^{D 2}$ (Fig. 4): the two projectors $\mathbb{P}^{D 1}$ and $\mathbb{P}^{D 2}$ share temporarily the same eigenvalue. At this moment, the two uni-dimensional modes join together in a bi-dimensional one of basis $\left(\mathbf{E}^{D 1}, \mathbf{E}^{D 2}\right)$ which leads (without demonstration) to a cubic symmetry revealed in Fig. 2 by its nine characteristic symmetry planes.

A general study of the symmetry group evolution would be of interest in a future work. The above analysis of the symmetry groups considers only exact symmetries. However, the evolution from a symmetry group to another is progressive. For example, on the pole figure at point $D$, the nine dark spots revealing the cubic symmetry are still perceptible but only the ones relative to the tetragonal symmetry are truly black (at the value 0 ). An animation of the pole figure between C' and D would reveal a progressive fading of the spots related symmetry planes which belong to the cubic symmetry but not to the orthogonal symmetry. Such distance between a stiffness tensor and a symmetry group can be measured (François et al., 1998; Moakher and Norris, 2006).

\subsection{Path dependance and non proportional loading}

The previous example (Sections 3.5, 4.3 and 5.5) was dedicated to two successive proportional loadings. On the contrary, we consider here two non proportional loadings:

$\boldsymbol{\varepsilon}^{\mathrm{I}}=\sin (\theta) \mathbf{B}^{1}-\cos (\theta) \mathbf{B}^{4}$
$\boldsymbol{\varepsilon}^{\mathrm{II}}=(1-\cos (\theta)) \mathbf{B}^{1}+\sin (\theta) \mathbf{B}^{4}$,

where $\theta$ varies between 0 and $\pi / 2$. 
Even if final strains are equals, the final stresses differ (Fig. 5) and this shows the history effect of the model. The deviatoric damage tensor evolves continuously and, at the final point, consists in a uni-dimensional weighted projector

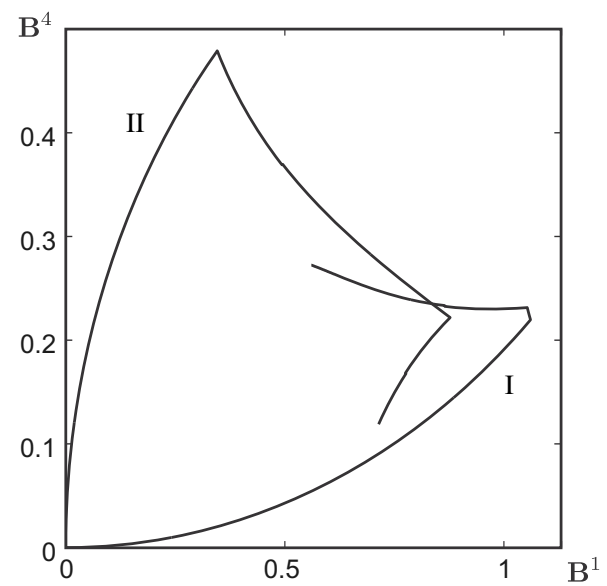

Fig. 5. Stress path for the two loadings I and II.
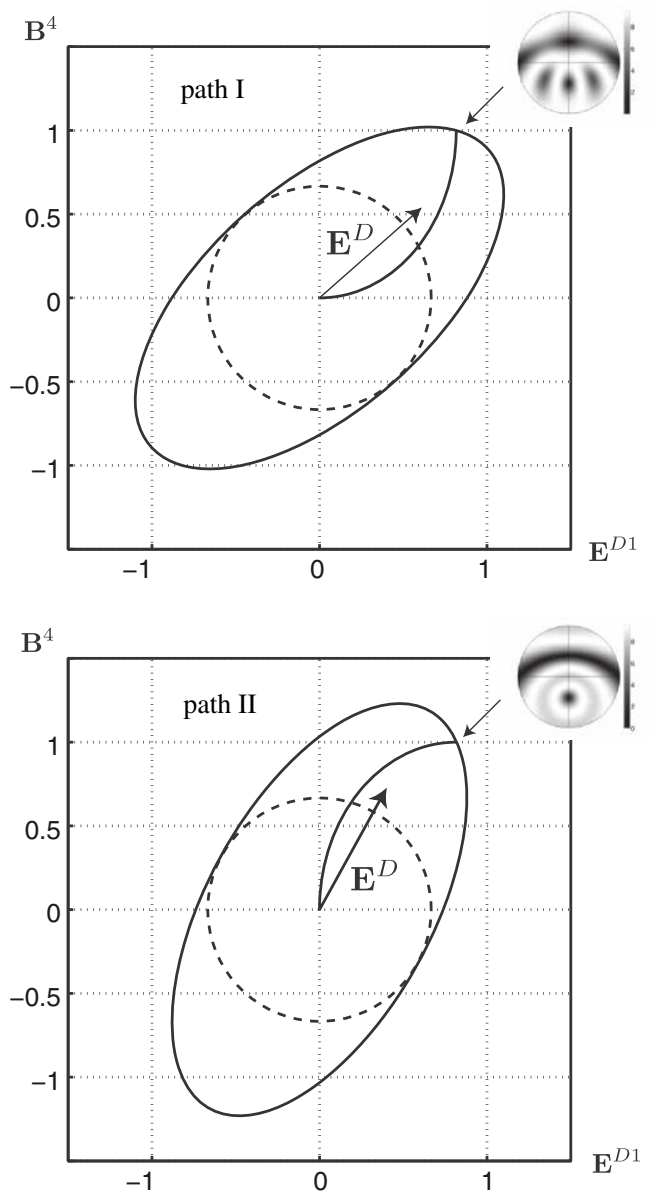

Fig. 6. Strain paths: initial (dashed) and last (plain) deviatoric yield surfaces.
$\mathbb{D}^{D I}=d^{D I} \mathbf{E}^{D I} \otimes \mathbf{E}^{D I}$ with $d^{D I}=0.75$ and $\mathbf{E}^{D I}=0.75 \mathbf{E}^{D 1}+0.66 \mathbf{B}^{4}$ for the path I and $\mathbb{D}^{D I I}=d^{D I I} \mathbf{E}^{D I I} \otimes \mathbf{E}^{D I I}$ with $d^{D I I}=0.76$ and $\mathbf{E}^{D I I}=0.48 \mathbf{E}^{D 1}+0.88 \mathbf{B}^{4}$ for the path II. The material is more damaged in tension for the loading $I$ and in shear for the loading II. These uni-dimensional projectors lead to yield surfaces of radius unchanged in the four (deviatoric) directions orthogonal to $\mathbf{E}^{D I}$ (respectively $\mathbf{E}^{D I I}$ ) as this can be observed (for one of them) on Fig. 6. The deviatoric tensors $\mathbf{E}^{D I}$ and $\mathbf{E}^{D I I}$ are orthotropic. However $\mathbb{D}^{D I}$ is accidentally close to be tetragonal and $\mathbb{D}^{D I I}$ close to be transverse isotropic (Fig. 6). These symmetry groups include the orthotropic one as allowed by the Curie principle.

\section{Conclusions}

The proposed damage model is consistent with both the thermodynamics of irreversible processes and the Kelvin eigentensor decomposition. In its present form it applies for the damage description of both initially isotropic and anisotropic materials, describing the continuous evolution of the symmetry group of the elasticity tensor within the respect of the Curie principle. The damage is represented by (fourth-rank) tensorial variables decomposed into their (second-rank) eigentensors which indicate the direction of damage and (scalar) eigenvalues which denote damage intensity. Due to the use of the Kelvin framework, the model is equivalently defined in term of stress or strain. Few material constants are required (a limit energy for each Kelvin mode). Each concept has a simple mechanical meaning. The model is already usable in a finite element code after the development of a more sophisticated integration scheme than the simple one used here.

The damage affects the response of the material in the tensorial direction of the actual eigenstrain and the present formulation induces (lower) damage recovery along the other (inactive) tensorial directions of the mode. This aspect is due to simplicity of the present exploratory model and further developments should include the introduction of coupling terms between active and passive damages, leaving the tensorial directions (thus the symmetry groups) unchanged with respect to the proposed model. In a similar manner, the retained energetic criterions are known (since the Beltrami criterion) to be quite unrealistic (especially for the non isochoric modes). Further enhancement may consist in the used of positive parts commonly used in damage mechanics, which have been shown to be consistent with the Kelvin's framework by Desmorat (2001).

\section{Appendix A}

Theorem 1. If $Q \in \mathcal{S O}(3)$ is an orthogonal transformation and $\mathbf{A}, \mathbf{B} \in \mathcal{T}$ then $Q(\mathbf{A}): Q(\mathbf{B})=\mathbf{A}: \mathbf{B}$.

Proof. In the canonical basis $\mathbf{e}^{i}$ :

$$
\begin{aligned}
Q(A)_{\mathrm{pq}} Q(B)_{\mathrm{pq}} & =Q_{\mathrm{pr}} Q_{\mathrm{qs}} A_{\mathrm{rs}} Q_{\mathrm{pm}} Q_{\mathrm{qn}} B_{\mathrm{mn}}=\delta_{\mathrm{rm}} \delta_{\mathrm{sn}} A_{\mathrm{rs}} B_{\mathrm{mn}} \\
& =A_{\mathrm{rs}} B_{\mathrm{rs}} .
\end{aligned}
$$

In particular, if $A=B$, this recalls that the Euclidean natural norm is preserved $Q(\mathbf{A}): Q(\mathbf{A})=\|Q(\mathbf{A})\|^{2}=\|\mathbf{A}\|^{2}$. 
Theorem 2. The matrix $\widehat{Q}_{p q}$ of an orthogonal transformation $Q$ in an orthonormal tensor basis $\mathbf{E}^{p}$ of $\mathcal{T}$ is orthogonal.

Proof. From theorem 1:

$$
\begin{aligned}
& Q\left(\mathbf{E}^{p}\right): Q\left(\mathbf{E}^{q}\right)=\delta_{p q}, \\
& \sum_{r, s=1}^{6} \widehat{Q}_{p r} \mathbf{E}^{r}: \widehat{Q}_{q s} \mathbf{E}^{s}=\sum_{r=1}^{6} \widehat{Q}_{p r} \widehat{Q}_{q r}=\delta_{p q} .
\end{aligned}
$$

This result can also be deduced from the Bond's expression of the matrix $\widehat{Q}_{q r}$ (Auld, 1973). It also applies in case of a transformation $Q^{i}$ internal in a subspace $\mathcal{T}^{i} \in \mathcal{T}$ (considering a basis $\mathbf{E}^{i p}$ of dimension $N^{i}$ of this subspace).

Theorem 3. The set of the orthogonal transformations $Q^{i}$ internal in $\mathcal{T}^{i}$ is the symmetry group of the projector $\mathbb{P}^{i}$ associated with $\mathcal{T}^{i}$.

Proof. Let $\mathbf{E}^{i p}$, where $i$ is fixed, be an orthonormal basis of $\mathcal{T}^{i}$ thus $\mathbb{P}^{i}=\sum_{p=1}^{N^{i}} \mathbf{E}^{i p} \otimes \mathbf{E}^{i p}$. Supposing $Q^{i}$ internal in $\mathcal{T}^{i}$, one has:

$Q^{i}\left(\mathbb{P}^{i}\right)=\sum_{p=1}^{N^{i}} Q^{i}\left(\mathbf{E}^{i p}\right) \otimes Q^{i}\left(\mathbf{E}^{i p}\right)=\sum_{p=1}^{N^{i}} \sum_{k=1}^{N^{i}} \sum_{r=1}^{N^{i}} \widehat{Q}_{p k}^{i} \widehat{Q}_{p r}^{i} \mathbf{E}^{i k} \otimes \mathbf{E}^{i r}$.

From the orthogonality of $\widehat{Q}^{i}$ (theorem 2):

$Q^{i}\left(\mathbb{P}^{i}\right)=\sum_{k=1}^{N^{i}} \mathbf{E}^{i k} \otimes \mathbf{E}^{i k}=\mathbb{P}^{i}$

thus $Q^{i}$ belongs to the symmetry group of $\mathbb{P}^{i}$.

To prove equivalence relation we suppose $\mathbb{P}^{i}=Q^{i}\left(\mathbb{P}^{i}\right)$ thus, for a given $r \in\left\{1 \ldots N^{i}\right\}$ :

$$
\begin{aligned}
& Q^{i}\left(\mathbf{E}^{i r}\right): \mathbb{P}^{i}: Q^{i}\left(\mathbf{E}^{i r}\right)=Q^{i}\left(\mathbf{E}^{i r}\right): Q^{i}\left(\mathbb{P}^{i}\right): Q^{i}\left(\mathbf{E}^{i r}\right), \\
& \sum_{q=1}^{N^{i}} Q^{i}\left(\mathbf{E}^{i r}\right): \mathbf{E}^{i q} \otimes \mathbf{E}^{i q}: Q^{i}\left(\mathbf{E}^{i r}\right) \\
& \quad=\sum_{p=1}^{N^{i}} Q^{i}\left(\mathbf{E}^{i r}\right): Q^{i}\left(\mathbf{E}^{i p}\right) \otimes Q^{i}\left(\mathbf{E}^{i p}\right): Q^{i}\left(\mathbf{E}^{i r}\right),
\end{aligned}
$$

and, from theorem 1:

$$
\sum_{q=1}^{N^{i}}\left(Q^{i}\left(\mathbf{E}^{i r}\right): \mathbf{E}^{i q}\right)^{2}=\sum_{p=1}^{N^{i}}\left(\mathbf{E}^{i r}: \mathbf{E}^{i p}\right)^{2}=1
$$

This proves that $Q^{i}\left(\mathbf{E}^{i r}\right) \in \mathcal{T}^{i}, \forall r \in\left\{1 \ldots N^{i}\right\}$ because its norm in $\mathcal{T}^{i}$ equals 1 (its norm in $\mathcal{T}$ ). Thus $Q^{i}$ is internal in $\mathcal{T}^{i}$ as envisaged.

Corollary 1. The symmetry group of a one dimensional projector $\mathbb{P}^{i}=\mathbf{E}^{i 1} \otimes \mathbf{E}^{i 1} \quad$ is $\quad\left\{Q \in \mathcal{S O}(3) / Q\left(\mathbf{E}^{i 1}\right)=\mathbf{E}^{i 1}\right\} \bigcup\{Q \in$ $\left.\mathcal{S O}(3) / Q\left(\mathbf{E}^{i 1}\right)=-\mathbf{E}^{i 1}\right\}$ (the first set is the symmetry group of $\left.\mathbf{E}^{i 1}\right)$.

Proof. From theorem 3 the symmetry group of $\mathbb{P}^{i}$ is the collection of orthogonal transformations $Q$ internal in $\mathcal{T}^{i}$. In this unidimensional subspace this includes the two possibilities $Q\left(\mathbf{E}^{i 1}\right)= \pm \mathbf{E}^{i 1}$.

\section{References}

Andrieux, S., Bamberger, Y., Marigo, J.J., 1986. A model of micro-cracked material for concretes and rocks. Journal de Mécanique 5, 471-513.

Annin, B.D., Ostrosablin, N.I., 2008. Anisotropy of elastic properties of materials. Journal of Applied Mechanics and Technical Physics 49, 998-1014.

Arramon, Y.P., Mehrabadi, M.M., Martin, D.W., Cowin, S.C., 2000. A multidimensional anisotropic strength criterion based on Kelvin modes. International Journal of Solids and Structures 37, 29152935.

Auffray, N., Bouchet, R., Bréchet, Y., 2009. Class-jump phenomenon for physical symmetries in bi-dimensional space. Lecture Notes in Applied and Computational Mechanics 46, 1-11.

Auld, B.A., 1973. Acoustic fields and waves in solids. John Wiley \& Sons.

Bazant, Z.P., 1984. Microplane model for strain controlled inelastic behaviour. Mechanics of Engineering Materials 43, 45-49.

Bazant, Z.P., Planas, J., 1998. Fracture and size effect in concrete and other quasi-brittle materials. CRC Press, New York.

Biegler, M.W., Mehrabadi, M.M., 1995. An energy-based constitutive damage model for anisotropic solids subject to damage. Mechanics of Materials 19, 151-164.

Boehler, J.P., Kirillov, A., Onat, E.T., 1994. On the polynomial invariants of elasticity tensors. Journal of Elasticity 34, 97-110.

Carpinteri, A., Chiaia, B., Cornetti, P., 2003. On the mechanics of quasibrittle materials with a fractal microstructure. Engineering fracture mechanics 70, 2321-2349.

Chaboche, J.L., 1988. Continuum damage mechanics, Part II: Damage growth, crack initiation, and crack growth. Journal of Applied Mechanics 55, 471-513.

Chalmers, A.F., 1970. Curie's principle. British Journal for the Philosophy of Science 21, 133-148.

Curie, P., 1894. Sur la symétrie dans les phénomènes physiques, symétrie d'un champ électrique et d'un champ magnétique. Journal of Physics and Théory and Applications 3, 393-415.

Desmorat, R., 2001. Dissymétrie de comportement élastique anisotrope couplé ou non á l'endommagement. C.R. Mécanique 328, 445-450.

Desmorat, R., 2009. Décomposition de Kelvin et concept de contraintes effectives multiples pour les matériaux anisotropes. C.R. Mécanique 337, 733-738.

Diner, C., Kochetov, M., Slawinski, M.A., 2011. Identifying symmetry classes of elasticity tensors using monoclinic distance function. Journal of Elasticity 102, 175-190.

Forte, S., Vianello, M., 1996. Symmetry classes for elasticity tensors. Journal of Elasticity 43, 81-108.

François, M., 1995. Identification des symétries matérielles de matériaux anisotropes. Thèse de doctorat. Université Pierre et Marie Curie Paris VI.

François, M., 2007. Behavior of cracked materials. Key Engineering Materials 349, 589-592.

François, M., Geymonat, G., Berthaud, Y., 1998. Determination of the symmetries of an experimentally determined stiffness tensor application to acoustic measurements. International Journal of Solids and Structures 35, 31-32.

Halm, D., Dragon, A., 1998. An anisotropic model of damage and frictional sliding for brittle materials. European Journal of Mechanics A/Solids 17, 439-460.

Halphen, B., Nguyen, Q.S., 1975. Sur les matériaux standards généralisés. Journal de Mécanique 14, 39-63.

He, Q.C., Zheng, Q.S., 1996. On the symmetries of 2D elastic and hyperelastic tensors. Journal of Elasticity 43, 203-225.

Helbig, K., 1994. Foundations of anisotropy for exploration seismics. Handbook of Geophysical Exploration, Section I. Seismic Exploration, vol. 22. Pergamon.

Hermann, C., 1934. Tensoren und Kristallsymmetrie. Zeitschrift für Kristallographie 80, 32-45.

Huo, Y., Del Piero, G., 1991. On the completeness of the crystallographic symmetries in the description of the symmetries of the elastic tensor. Journal of Elasticity 25, 203-246.

Kachanov, L.M., 1958. On the time to failure under creep conditions. Izvestija Akademii Nauk SSR, Otdelenie tekhnicheskikh Nauk 8, 2631.

Ladevèze, P., 1993. On anisotropic damage theory, Failure criteria of structured media, In: Proceedings of the CNRS international colloquium No 351, pp. 355-363.

Lemaitre, J., 1996. A Course on Damage Mechanics. Springer, Heidelberg.

Matallah, M., La Borderie, C., 2009. Inelasticity-damage based model to numerical modeling of concrete cracking. Engineering Fracture Mechanics 76, 1087-1108. 
Mattei, N.J., Mehrabadi, M.M., Zhu, H., 2007. A micromechanical constitutive model for the behavior of concrete. Mechanics of Materials 39, 357-379.

Mazars, J., Pijaudier-Cabot, G., 1996. From damage to fracture mechanics and conversely: a combined approach. International Journal of Solids and Structures 33, 3327-3342.

Mehrabadi, M.M., Cowin, S., 1990. Eigentensors of linear anisotropic elastic materials. Quarterly Journal of Mechanics and Applied Mathematics 43, 15-41.

Moakher, M., Norris, A.N., 2006. The closest elastic tensor of arbitrary symmetry to an elasticity tensor of lower symmetry. Journal of Elasticity 85, 215-263.

Onat, E.T., 1984. Effective properties of elastic materials that contain penny shaped voids. International Engineering and Science 22, 10131021.

Ostrosablin, N.I., 1984. On the structure of the elasticity moduli tensor: elastic eigenstates, in: Dynamics of Continuous Media (in Russian). Acad. Sci. USSR, Novosibirsk. vol. 66, pp. 113-125.

Pensée, V., Kondo, D., Dormieux, L., 2002. Micromechanical analysis of anisotropic damage in brittle materials. Journal of Engineering and Mechanics ASCE 128, 889-897.

Raous, M., Cangémi, L., Cocu, M., 1998. A consistent model coupling adhesion, friction and unilateral contact. Computing Methods and Applied Mechanics and Engineering 177, 383-3997.

Renaud, P., 1935. Sur une généralisation du principe de symétrie de Curie. Comptes Rendus De L Academie Des Sciences 200, 531-534.
Rosen, J., 1982. Symmetries in Physics: selected reprints. American Association of Physics Teachers, New York.

Rosen, J., 1995. Symmetry in Science: An Introduction to the General Theory. Springer-Verlag, New York.

Royer-Carfagni, G., Salvatore, W., 2000. A micromechanical constitutive model for the behavior of concrete. Mechanics of Cohesive-frictional Materials 5, 535-563.

Rychlewski, J., 1984. On Hooke's law. Prikladnaya Matematika Mekhanika 48, 303-314.

Schreyer, H.L., 1995. Continuum damage based on elastic projection operators. International Journal of Damage Mechanics 4, 171-195.

Schreyer, H.L., Zuo, Q.H., 1995. Anisotropic yield surfaces based on elastic projection operators. ASME Journal of Applied Mechanics 62, 780785.

Sivardière, J., 1995. La symétrie en mathématiques, physique et chimie. PUG, Grenoble.

Thomson (Lord Kelvin), W., 1856. Elements of a mathematical theory of elasticity. Philosophical Transactions of the Royal Society of London 146, 481-498.

Xiao, H., Bruhns, O.T., Meyers, A., 2006. On isotropic extension of anisotropic constitutive functions via structural tensors. Zeitschrift Fur Angewandte Mathematik Und Mechanik 86, 151-161.

Zheng, Q.S., Boehler, J.P., 1994. The description, classification, and reality of material and physical symmetries. Acta Mechanica 102, 73-89. 